\title{
Ultraspectral sounding retrieval error budget and estimation
}

\author{
Daniel K. Zhou*1, Allen M. Larar ${ }^{1}$, Xu Liu ${ }^{1}$, William L. Smith ${ }^{2,3}$, \\ L. Larrabee Strow ${ }^{4}$, and Ping Yang ${ }^{5}$ \\ ${ }^{1}$ NASA Langley Research Center, Hampton, VA, USA \\ ${ }^{2}$ Hampton University, Hampton, VA, USA \\ ${ }^{3}$ University of Wisconsin-Madison, Madison, WI, USA \\ ${ }^{4}$ University of Maryland Baltimore County, Baltimore, MD, USA \\ ${ }^{5}$ Texas A\&M University, College Station, TX, USA
}

\begin{abstract}
The ultraspectral infrared radiances obtained from satellite observations provide atmospheric, surface, and/or cloud information. The intent of the measurement of the thermodynamic state is the initialization of weather and climate models. Great effort has been given to retrieving and validating these atmospheric, surface, and/or cloud properties. Error Consistency Analysis Scheme (ECAS), through fast radiative transfer model (RTM) forward and inverse calculations, has been developed to estimate the error budget in terms of absolute and standard deviation of differences in both spectral radiance and retrieved geophysical parameter domains. The retrieval error is assessed through ECAS without assistance of other independent measurements such as radiosonde data. ECAS re-evaluates instrument random noise, and establishes the link between radiometric accuracy and retrieved geophysical parameter accuracy. ECAS can be applied to measurements of any ultraspectral instrument and any retrieval scheme with associated RTM. In this paper, ECAS is described and demonstration is made with the measurements of the METOP-A satellite Infrared Atmospheric Sounding Interferometer (IASI).
\end{abstract}

Keywords: Atmospheric composition, error, radiative transfer, remote sensing, satellite.

\section{INTRODUCTION}

The ultraspectral infrared radiances obtained from satellite observations provide atmospheric, surface, and/or cloud information. The intent of the measurement of the thermodynamic state is the initialization of weather and climate models. Great effort has been given to retrieving these atmospheric, surface, and/or cloud properties using advanced retrieval algorithms developed with fast radiative transfer models (RTMs), including cloud effects. Validation efforts must be given to calibrated radiances and retrieval geophysical parameters ${ }^{1-3}$. For example, Tobin et al. (2006) used a multi-instrument/platform correlative measurement dataset to build the best-estimated atmospheric state for each individual satellite measurement for validation. Pougatchev et al. (2009) have developed a linear statistical Validation Assessment Model (VAM) providing the best estimated atmospheric state and corresponding nominal satellite measurement using the correlative data per se. These validation methods are accurate, but depend on other independent measurements, such as radiosonde data, and the accuracy of these independent "coincident" measurements. These data usually were collected during dedicated field campaigns and/or matchup soundings (e.g., radiosonde and Raman Lidar data). In practice, the previous validation studies are more complex in considering that "coincident" measurement or that measurement-derived "truth" is at the same location and time. In addition, the vertical and horizontal (i.e., footprint size) resolutions of other independent measurements have to be taken into account as well. The instrumental averaging kernels are typically used to resolve the difference in vertical resolution. However, the difference of horizontal footprint size is too complex to consider and often neglected.

Our motivation for this current work is to understand and estimate the retrieval error contributed by major error sources in obtaining a link between the retrieved-geophysical-parameters and the radiometric accuracies. These errors are (1) retrieval errors from ill-posed retrieval system that include but not limit smoothing errors, (2) retrieval noise from

*daniel.k.zhou@nasa.gov, Tel: 757-864-5663 
instrument random noise, and (3) "un-modeled" errors from the discrepancy between the RTM retrieval-simulated and measured radiances. We would like to answer questions such as how the radiometric random noise in the measurement transfer relates to the error (or retrieval noise) in the retrieved parameters, e.g., temperature and moisture profiles. What is the retrieval error introduced by an ill-posed retrieval model? We have developed a statistical Error Consistency Analysis Scheme (ECAS) through fast RTM forward and inverse calculations to estimate the error budget in terms of bias and standard deviation of differences (i.e., STDE) in both spectral radiance and retrieved-geophysical-parameter domains. This scheme provides an internal consistency check with RTM and $\mathrm{RTM}^{-1}$ calculations to establish a reliable link between radiometric error in the spectral radiance domain and retrieval error in the geophysical-parameter domain. This scheme limits the uncertainty introduced by the different time and space, which is critically important in previous validation studies ${ }^{2,3}$. The measurements are emulated with known geophysical parameters, identified error sources like the random noise level, and the discrepancy between the RTM simulated and measured radiances. The error and/or uncertainty of each retrieved parameter is given by ECAS analysis, providing detailed information on the quality of retrieved products in reproducing the radiances equivalent to the measurements. The retrieval error budget estimation within this study is investigated in both radiance and retrieved-geophysical-parameter domains. ECAS is described in Section 2 and initial demonstration is given in Section 3 using the measurements of the Infrared Atmospheric Sounding Interferometer (IASI) on the METOP-A satellite ${ }^{4,5}$ during the Joint Airborne IASI Validation Experiment (JAIVEx) April to May $2007^{1,6}$.

\section{METHODOLOGIES}

ECAS is to estimate retrieval error through a closed-loop iterated geophysical parameter retrieval and radiance simulation with a set of observations. Sensor-calibration-estimated random noise is initially used in the analysis to estimate a realistic, random noise from this set of observations. The analysis can be applied to any RTM and its inversion (hereafter denoted as $\mathrm{RTM}^{-1}$ ). It is noted that we assume all error sources are independent; also, the one-dimensional, variational (1-D Var.) retrieval presented herein may not be optimal because an estimated error is used for all retrievals. Retrievals from measured radiance may contain an error slightly different from retrieval-simulated radiance because training representation is optimal for retrieval-simulated radiance but not for real data. Nevertheless, we believe that these effects are small enough to be ignored. A detailed analysis flowchart summarizing the methodology and analysis procedure is given in Figure 1. The notations listed in the figure are used in the text below. As indicated, only a set of observed radiances $\left(R_{m}\right)$ and its calibration-estimated instrument random noise $\left(\delta_{c}\right)$ are needed.
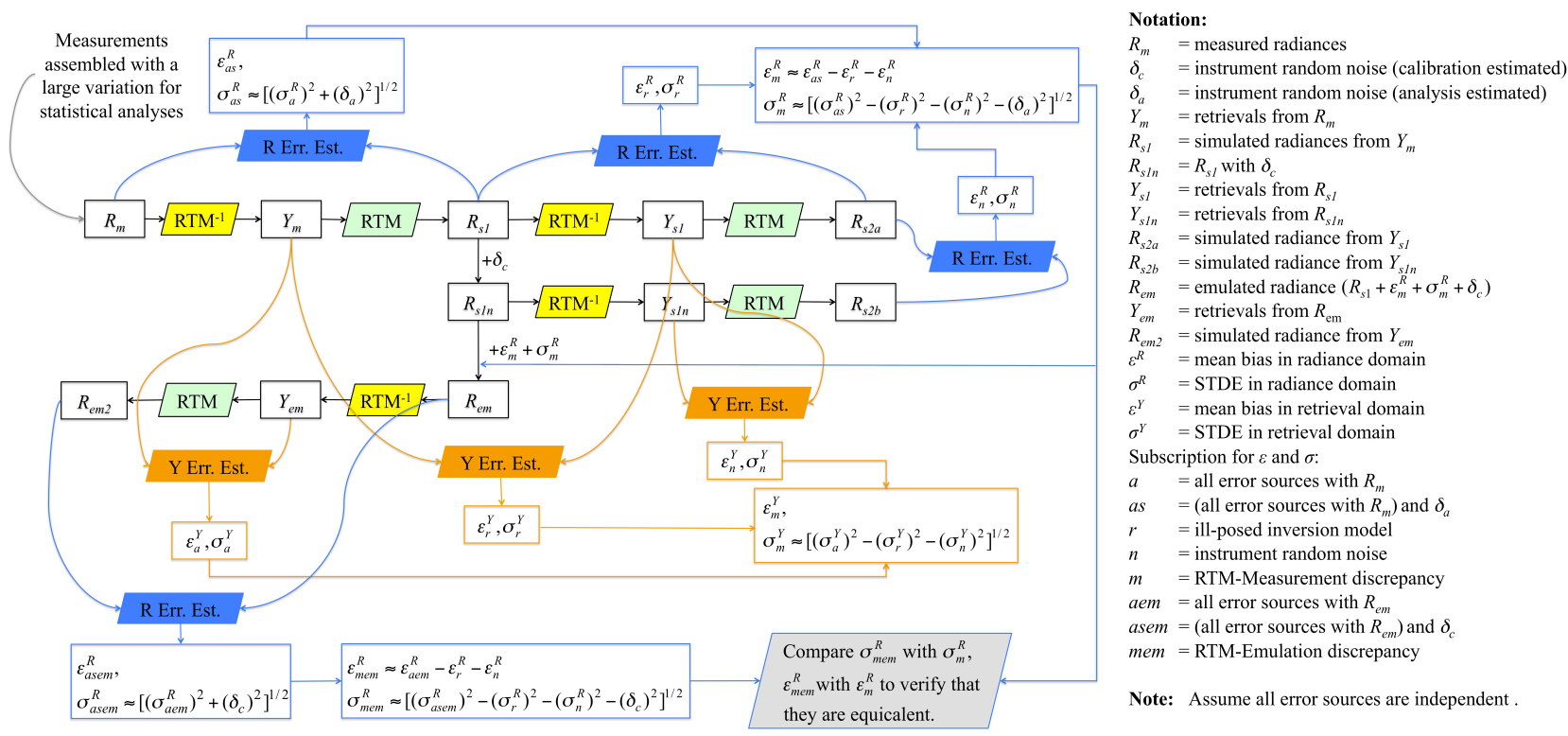

Fig. 1. Error Consistency Analysis Scheme (ECAS) and analysis flowchart. 
Here, we demonstrate this method and its outcomes using IASI measurements. The retrieval algorithm $\left(\mathrm{RTM}^{-1}\right)^{6,7}$ used here for demonstration only uses measured radiance and instrument noise; no other "truth" data from satellite or surfacebased instruments or from numerical weather analysis/prediction models is utilized in assisting or constraining the retrieval products. The fast transmittance model used herein is a combination of the Stand-alone AIRS Radiative Transfer Algorithm (SARTA) Version 1.07 and the physically-based cloud RTM is based on the DIScrete Ordinate Radiative Transfer (DISORT) calculations performed for a wide variety of cloud microphysical properties ${ }^{8-10}$. An iterative, one-dimensional, variational (1-D Var.), multi-variable inversion using the minimum-information regularization method is used for obtaining the final retrieval. An all-season, global EOF regression database is used to obtain the initial profile for the 1-D Var. physical retrieval. The physical iterative retrieval can effectively, but not fully, account for non-linearity and further improves retrieval accuracy from the initial profile produced by EOF linear regression. The regression coefficients are classified with respect to cloud-free and cloudy conditions. The algorithm and details are found elsewhere ${ }^{6,7}$. The weighting functions can be calculated by an analytical scheme or a numerical perturbation method. The weighting functions associated with water vapor and constant mixing ratio gases have peaks distributed throughout the range of pressure altitude. In other words, these spectral channels can be used for temperature and water vapor profile retrieval.

\section{ANALYSIS DEMONSTRATION}

This analysis can be applied to any ultraspectral measurements from instruments, such as the aircraft instruments NAST-I and SHIS, and the satellite instruments AIRS, IASI, and CrIS. Here we use IASI measurements, and the data used herein were collected during the JAIVEx campaign. Four granules, from 19 April, 27 April, 29 April, and 04 May 2007, are used in the analysis. Each granule contains 5400 measurements covering the same area of the continental US and the Gulf of Mexico. Only retrievals identified as "clear-sky" measurements are used to represent error budget estimations under "clear-sky" conditions. A detailed case study of April 29, 2007, inter-comparison between IASI, AIRS, and NAST-I retrievals, as well as dedicated radiosonde measurements, can be found elsewhere ${ }^{6}$; it is worthwhile to note that the same retrieval algorithm is used in this study.

Spectral radiance discrepancy between RTM calculation and observation is derived. Demonstration shown in Figures 2-5 is from a granule of 2007.04.19 (nighttime) over the continental US, using 3398 "clear" cases out of a total of 5400 observations. Figure 2a, derived from noise-free simulated radiance and measured noisy radiance, plots the $\sigma_{a s}^{R}$, and can be express as $\sigma_{a s}^{R}=\left[\left(\sigma_{a}^{R}\right)^{2}+\left(\delta_{a}\right)^{2}\right]^{1 / 2}$. In order to estimate $\sigma_{m}^{R}$, we need to know $\sigma_{r}^{R}, \sigma_{n}^{R}$, and a realistic instrument random noise measurement of this data set $\delta_{a}$. Both $\sigma_{r}^{R}$ and $\sigma_{n}^{R}$ can be estimated through simulations (see Figure 1). Now $\delta_{a}$ is estimated by scaling the calibration-estimated instrument noise $\delta_{c}$ down (see Figure $2 \mathrm{~b}$ ) and making sure that derived $\sigma_{m}^{R}$ is reasonably consistent with that $\sigma_{a s}^{R} \geq\left[\left(\sigma_{r}^{R}\right)^{2}+\left(\sigma_{n}^{R}\right)^{2}+\delta_{a}^{2}\right]^{1 / 2}$ (i.e., $\left.\sigma_{m}^{R} \geq 0\right)$, and can be verified later on using $\delta_{a} \approx\left[\left(\sigma_{a s}^{R}\right)^{2}-\left(\sigma_{r}^{R}\right)^{2}-\left(\sigma_{n}^{R}\right)^{2}-\left(\sigma_{m e m}^{R}\right)^{2}\right]^{1 / 2}$. In other words, it will be verified that $\sigma_{m e m}^{R}$ is equivalent to $\sigma_{m}^{R}$. Figure $2 \mathrm{~b}$ plots calibration-estimated ( $\delta_{c}$ in magenta), realistic instrument noise of this data set ( $\delta_{a}$ in blue), and the spectral STDE introduced by random noise and ill-posed retrieval $\left\{\sigma_{r+n}^{R}=\left[\left(\sigma_{r}^{R}\right)^{2}+\left(\sigma_{n}^{R}\right)^{2}\right]^{1 / 2}\right.$ in red $\}$. Now the STDE and bias in the spectral domain plotted in Figures $3 \mathrm{a}$ and $3 \mathrm{~b}$, respectively, are estimated with three components contributed by ill-posed retrieval, instrument random noise, and the discrepancy between the simulated and measured radiances.

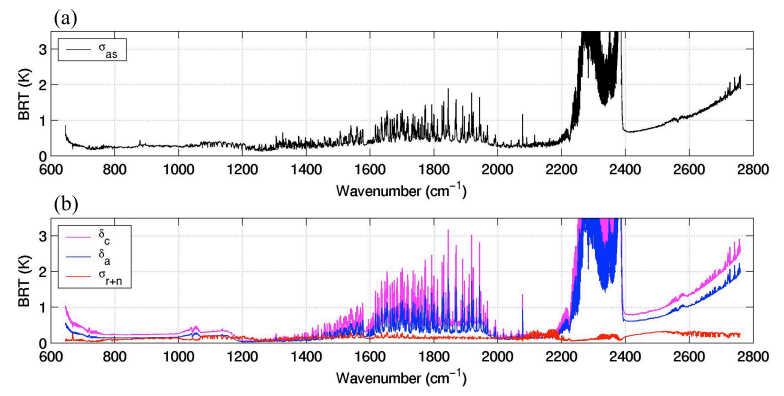

Fig. 2. (a) Total estimated STDE using retrieval simulated and measured radiance, (b) calibration-estimated (in magenta), realistic instrument noise (in blue), and the spectral STDE introduced by random noise and ill-posed retrieval (in red). 

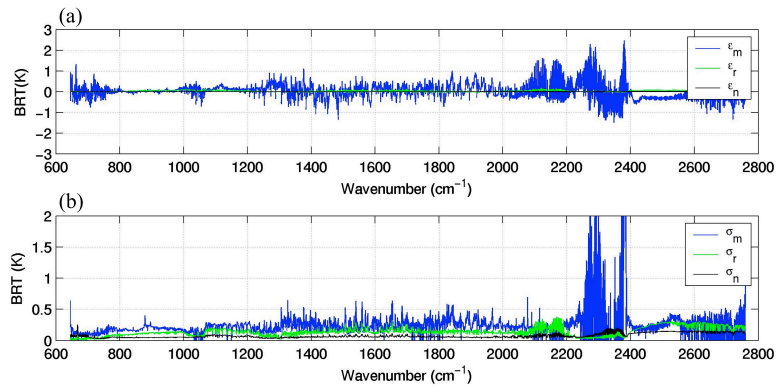

Fig. 3. (a) Bias and (b) STDE in radiance domain contributed from three error sources (see text).

Following the ECAS flowchart of Figure 1, a so-called "measured" radiance is emulated $\left(R_{e m}\right)$ by adding instrument noise $\left(\delta_{c}\right)$ and the discrepancy between the retrieval-simulated and measured radiances $\left[\varepsilon_{m}^{R}, \sigma_{m}^{R}\right]$, over noise-free radiance $\left(R_{s I}\right)$ simulated from the retrieval $\left(Y_{m}\right)$ associated with the measurement $\left(R_{m}\right)$. Figure 4 plots the total error estimated using retrieval simulated and emulated radiances $\left(\sigma_{\text {asem }}^{R}\right)$, which is used together with $\delta_{c}$ and $\sigma_{r+n}^{R}$ (shown in Figure $2 \mathrm{~b}$ ) to estimate the discrepancy between the noise-free model and emulated noisy radiances $\left[\varepsilon_{\text {mem }}^{R}, \sigma_{m e m}^{R}\right]$ that are compared with that derived from the measurements $\left[\varepsilon_{m}^{R}, \sigma_{m}^{R}\right]$. The comparison shown in Figures $5 \mathrm{a}$ and $5 \mathrm{~b}$, verifies that the radiance error derived from emulated radiance is similar to that derived from measured radiance.

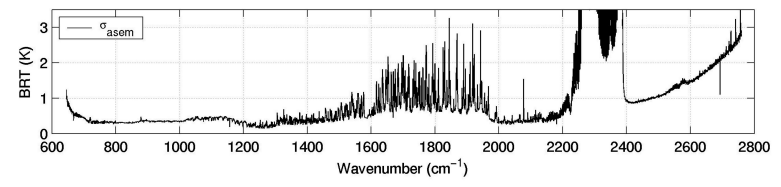

Fig. 4. Total estimated STDE using retrieval simulated and emulated radiance.
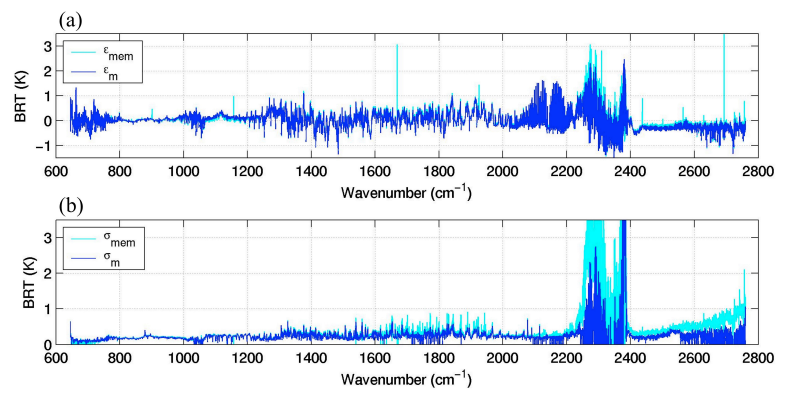

Fig. 5. "Un-modeled" error: (a) bias and (b) STDE in radiance domain introduced by the measured (in blue) and emulated radiance (in cyan).

The purpose of emulating measured-like radiances from a known geophysical state is to estimate the total error in retrieval domain. Since the total error between $Y_{m}$ and $Y_{e m}$ is caused by (1) the instrument noise $\delta_{c}$, (2) the discrepancy between simulated and measured radiances $\left[\varepsilon_{m}^{R}, \sigma_{m}^{R}\right]$, and (3) of course the retrieval system itself. Now the error $\left[\varepsilon_{m}^{Y}, \sigma_{m}^{Y}\right]$, induced by the discrepancy between simulated and measured radiances can be estimated by the total error subtracting the error induced by the instrument noise and ill-posed retrieval problem. The ill-posed retrieval error and instrument-noiseinduced error in the retrieval domain is straightforward, as shown in Figure 1. The temperature and moisture profile errors (i.e., bias and STDE) in a relative form to its profile are plotted in Figures 6 and 7. Retrieval error estimations shown in Figures 6 and 7 are derived from four granules [of 2007.04.19 (nighttime), 04.27 (daytime), 04.29 (daytime), and 05.04 (daytime)] over the continental US and the Gulf of Mexico using 11871 identified "clear" cases out of a total 21600 observations. For example, if a temperature relative STDE is 0.12 at $5 \mathrm{~km}$, and the temperature is at $275 \mathrm{~K}$, then the temperature STDE will be $0.33 \mathrm{~K}$. Retrieval errors of surface parameters (e.g., surface skin temperature and emissivity) from the same dataset are computed in the same manner. For surface skin temperature $\left(T_{s}\right)$, the errors 
contributed by different sources $\left[\varepsilon_{n}^{T_{s}}, \sigma_{n}^{T_{s}}\right],\left[\varepsilon_{r}^{T_{s}}, \sigma_{r}^{T_{s}}\right]$, and $\left[\varepsilon_{m}^{T_{s}}, \sigma_{m}^{T_{s}}\right]$ are $[0.003,0.255],[-0.119,0.288]$, and $[-0.142,0.181]$, respectively, in Kelvin degrees. The total $T_{s}$ errors $\left[\varepsilon_{a}^{T_{s}}, \sigma_{a}^{T_{s}}\right]$ are estimated as $[-0.258,0.421]$ in Kelvin degrees. The surface emissivity error for this dataset is also estimated and they are in the order of $10^{-3}$ and below. It is noted that the error estimation for other retrieved parameters is not illustrated here but performed in the same manner. It is noted that the instrument random noise $\delta_{c}$ can be replaced by the analysis estimated random noise $\delta_{a}$ to obtain retrieval noise error $\left[\varepsilon_{n}^{Y}, \sigma_{n}^{Y}\right]$ from $\delta_{a}$. This step is omitted and $\left[\varepsilon_{n}^{Y}, \sigma_{n}^{Y}\right]$ from $\delta_{c}$ reported herein (Figures 6 and 7) might be slightly overestimated as $\delta_{c}>\delta_{a}$ for this case. The difference should be minimal.
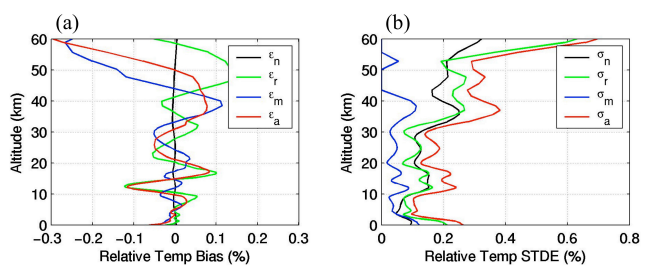

Fig. 6. Retrieved temperature profile error budget estimation. Relative value is (error/profile) $\times 100$.
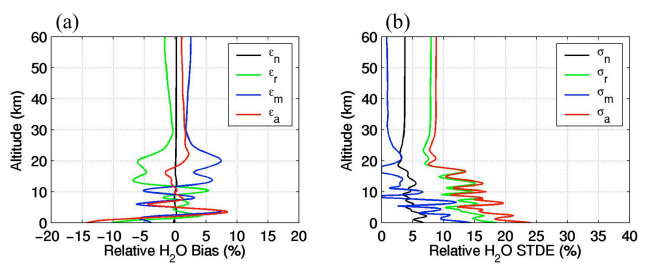

Fig. 7. Same as Fig. 6 but for moisture

\section{DISCUSSION AND SUMMARY}

The discrepancy between the RTM retrieval-simulated and measured radiances can be different from time to time and depends on the atmospheric conditions (e.g., un-modeled error from effects of aerosol, dust, and other trace species). The instrument noise may also vary from time to time. The advantages of this analysis are that we can effectively estimate the instrument random noise of the dataset and the error budget from our RTM and $\mathrm{RTM}^{-1}$ systems in both radiance domain and retrieval domain. Accurate estimations of $\left[\varepsilon_{m}^{R}, \sigma_{m}^{R}\right]$ and $\delta_{a}$ might be updated in a 1-D Var. retrieval system to improve the retrieval. Since the iteration between radiance simulations and retrievals starts with measured radiance spectra, the horizontal footprint size of a retrieved profile obtained from its associated-measured spectrum is the same as the one it compares with to produce the final retrieval error. The retrieval errors resulting from this ill-posed retrieval system, $\left[\varepsilon_{r}^{Y}, \sigma_{r}^{Y}\right]$, are computed from retrievals $Y_{m}$ and $Y_{s l}$. These retrievals, produced with the same retrieval system and very similar radiances, have a very similar but not same vertical resolution. There is no time and space difference between the profiles used for comparison. This provides a critical advantage of limiting the uncertainty or so-called "artificial smoothing error" caused by a different time and space of the "truth" in retrieval validation or error estimation. It is worth to pointing out that the weakness of the ECAS is still an "ill-posed" problem with which we are dealing, given the retrieval parameters and their errors compensating to a certain minimal degree among themselves, although constraints are used in the retrieval to minimize such cross talk among retrieved parameters. For example, the retrieval errors estimated herein for surface skin temperature and emissivity spectrum could compensate each other to satisfy a minimal radiance fitting in the retrieval process.

\section{ACKNOWLEDGEMENTS}

This research is supported by NASA Langley Research Center and NASA Headquarters. IASI was developed and built under the responsibility of the Centre National d'Etudes Spatiales (CNES). It is flown aboard the METOP satellites as part of the EUMETSAT Polar System. The IASI L1C data are received through the Unified Meteorological Archival and Retrieval Facility (UMARF) of EUMETSAT. 


\section{REFERENCES}

1. A. M. Larar, W. L. Smith, D. K. Zhou, X. Liu, H. Revercomb, J. P. Taylor, S. M. Newman, and P. Schlüssel, "IASI spectral radiance validation inter-comparisons: case study assessment from the JAIVEx field campaign," Atmos. Chem. Phys., 10, 441-430 (2010) [doi:10.5194/acp-10-411-2010].

2. D. C. Tobin, H. E. Revercomb, R. O. Knuteson, F. A. Best, W. L. Smith, N. N. Ciganovich, R. G. Dedecker, S. Dutcher, S. D. Ellington, R. K. Garcia, H. B. Howell, D. D. LaPorte, S. A. Mango, T. S. Pagano, J. K. Taylor, P. van Delst, K. H. Vinson, and M. W. Werner, "Radiometric and spectral validation of Atmospheric Infrared Sounder observations with the aircraft-based Scanning High-Resolution Interferometer Sounder," J. Geophys. Res., 111, D09S02 (2006) [doi:10.1029/2005JD006094].

3. N. Pougatchev, T. August, X. Calbet, T. Hultberg, O. Oduleye, P. Schlüssel, B. Stiller, S. K. Germain, and G. Bingham, "IASI temperature and water vapor retrievals - error assessment and validation," Atmos. Chem. Phys., 9, 6453-6458 (2009) [doi:10.5194/acp-9-6453-2009].

4. D. Blumstein, G. Chalon, T. Carlier, C. Buil, P. Hebert, T. Maciaszek, G. Ponce, and T. Phulpin, "IASI instrument: technical overview and measured performances," SPIE Proc., 5543, 196-207 (2004) [doi:10.1117/12.560907].

5. D. K. Klaes, M. Cohen, Y. Buhler, P. Schlüssel, R. Munro, J.-P. Luntama, A. Von Engeln, E. Ó. Clerigh, H. Bonekamp, J. Ackermann, J. Schmetz, “An introduction to the EUMETSAT Polar System”, B. Am. Meteorol. Soc., 88, 1085-1096 (2007) [doi:10.1175/BAMS-88-7-1085].

6. D. K. Zhou, W. L. Smith, A. M. Larar, X. Liu, J. P. Taylor, P. Schlüssel, L. L. Strow, and S. A. Mango, “All weather IASI single field-of-view retrievals: case study - validation with JAIVEx data," Atmos. Chem. Phys., 9, 2241-2255 (2009) [doi:10.5194/acp-9-2241-2009].

7. D. K. Zhou, W. L. Smith, Sr., X. Liu, A. M. Larar, S. A. Mango, and H.-L. Huang, "Physically retrieving cloud and thermodynamic parameters from ultraspectral IR measurements," J. Atmos. Sci., 64, 969-982 (2007) [doi:10.1175/JAS3877.1].

8. L. L. Strow, S. E. Hannon, S. De Souza-Machado, H. E. Motteler, and D. Tobin, "An overview of the AIRS radiative transfer model," IEEE Trans. Geosci. Remote Sens., 41, 303-313 (2003) [doi:10.1109/TGRS.2002.808244].

9. K. Stamnes, S.-C. Tsay, W. Wiscombe, and K. Jayaweera, "Numerically stable algorithm for discrete-ordinatemethod radiative transfer in multiple scattering and emitting media," App. Opt., 27, 2502-2509 (1988) [doi:10.1364/AO.27.002502].

10. P. Yang, B. C. Gao, B. A. Baum, Y. Hu, W. J. Wiscombe, S.-C. Tsay, D. M. Winker, and S. L. Nasiri, "Radiative Properties of cirrus clouds in the infrared (8-13 $\mu \mathrm{m})$ spectral region," J. Quant. Spectros. Radiat. Transfer, 70, 473504 (2001) [doi:10.1016/S0022-4073(01)00024-3]. 